\title{
Post-Diagnostic Beta Blocker Use and Breast Cancer-Specific Mortality: A Population-Based Cohort Study
}

\section{Oliver William Scott ( $\square$ o.scott@auckland.ac.nz )}

The University of Auckland https://orcid.org/0000-0002-8994-2760

\section{Sandar TinTin}

University of Auckland

J Mark Elwood

University of Auckland

Alana Cavadino

University of Auckland

Laurel A Habel

Kaiser Permanente Northern California

Marion Kuper

Waikato District Health Board

lan Campbell

Waikato District Health Board

\section{Ross Lawrenson}

University of Waikato

\section{Research Article}

Keywords: breast cancer, mortality, beta blockers, pharmacoepidemiology, cohort study

Posted Date: December 3rd, 2021

DOI: https://doi.org/10.21203/rs.3.rs-1106678/v1

License: (1) (1) This work is licensed under a Creative Commons Attribution 4.0 International License.

Read Full License

Version of Record: A version of this preprint was published at Breast Cancer Research and Treatment on March 14th, 2022. See the published version at https://doi.org/10.1007/s10549-022-06528-0. 


\section{Abstract}

Purpose Beta blockers (BB) have been associated with improved, worsened, or unchanged breast cancer outcomes in previous studies. This study examines the association between the post-diagnostic use of BBs and death from breast cancer in a large, representative sample of New Zealand (NZ) women with breast cancer.

Methods Women diagnosed with a first primary breast cancer between 2007 and 2016 were identified from four population-based regional NZ breast cancer registries and linked to national pharmaceutical data, hospital discharges, and death records. The median follow up time was 4.51 years. Cox proportional hazard models were used to estimate the hazard of breast cancer-specific death (BCD) associated with post-diagnostic BB use.

Results Of the 14,976 women included in analyses, $21 \%$ used a BB after diagnosis. BB use (vs non-use) was associated with a small and non-statistically significant increased risk of BCD (adjusted hazard ratio: $1.11 ; 95 \% \mathrm{Cl}$ : 0.95-1.29). A statistically significant increased risk confined to short-term use (0-3 months) was seen ( $H R=1.40 ; 1.14-1.73)$, and this risk steadily decreased with increasing duration of use and became a statistically significant protective effect at $3+$ years of use (HR=0.54; $0.34-0.87)$.

Conclusion Our findings suggest that any increased risk associated with BB use may be driven by risk in the initial few months of use. Long-term BB use may be associated with a reduction in BCD.

\section{Introduction}

Breast cancer is the most common cancer in women and the leading cause of female cancer mortality worldwide [1]. Comorbidities are common in breast cancer patients [2], and there is a high and increasing prevalence of risk factors for both breast cancer and ischemic heart disease among western women [3-5]. As such, many breast cancer patients use prescribed medications for cardiovascular conditions. Examining the association between commonly used cardiovascular medications and breast cancer outcomes is therefore warranted. Beta blockers (BBs), principally indicated for angina, arrhythmias, heart failure, hypertension, and myocardial infarction [6, 7], are commonly used cardiovascular medications that have been used in Western medicine for decades [8-10].

Human breast cancer cells have beta-adrenergic receptors [11]. Responses induced by beta-adrenergic signalling include upregulated expression of metastasis-associated genes involved in inflammation, angiogenesis, and tissue invasion, and downregulated expression of genes facilitating anti-tumour immune responses [12]. Beta-adrenergic receptors mediate the catecholamine hormones produced in the stress response, which in turn produce the aforementioned responses [13]. These hormones can be blocked by BBs, resulting in a potential protective effect through interference with tumour cell proliferation and migration, as well as tumoural angiogenesis $[14,15]$. On the basis of this evidence, several observational studies have been carried out, and some have reported that BB use may be protective for breast cancer-specific death (BCD) [16-19]. However, others have noted no association 
between BBs and BCD [20-25], and one reported BBs were associated with an increased risk of BCD [26]. The only RCT carried out on BBs (to date) found that preoperative propranolol downregulated biomarkers of invasive potential and inflammation, and improved biomarkers of cellular immune response [27].

There have been several methodological limitations in previous studies examining the association between BB use and BCD. For example, some studies fail to account for the time varying nature of medication use [28]. Moreover, there have been few studies which have explored the dose-response effect of BBs. Therefore, our primary objective was to address these limitations and explore the relationship between post-diagnostic BB use and BCD in a large population-based cohort study of newly diagnosed breast cancer patients in New Zealand.

\section{Methods}

\section{Data sources}

Eligible women were all those with a first primary breast cancer diagnosed and recorded in any of four population-based regional breast cancer registries (Auckland, Waikato, Wellington, and Christchurch) [29] in New Zealand between 1 Jan 2007 and 31 Dec 2016. These registers include all women diagnosed with breast cancer in their defined areas, and together cover about $70 \%$ of all breast cancer registrations in New Zealand. Using an anonymised National Health Index number, data were linked to several national data bases: the Pharmaceutical Collection (PHARMS), a national database containing dispensing information and medication identifiers from pharmacists for subsidised dispensings [30]; the National Minimum Dataset, relating to all day patients and inpatients discharged from both public and private hospitals; and the National Mortality Collection, with information about all certified deaths [31]. Women were excluded if their records did not link to at least one dispensing from the pharmaceutical collection $(n=14)$ or if their date of death was on or before their recorded date of breast cancer diagnosis $(n=3)$. The final cohort for analyses was comprised of 14,976 women. This study was approved by the Central Health and Disability Ethics Committee (Ref: 19/CEN/4).

\section{Exposure and outcome data}

In the PHARMS database, medications dispensed after breast cancer diagnosis were determined using the therapeutic group ID, a PHARMAC identifier for each group of Anatomical, Therapeutic, and Chemical properties [32]. All BBs were included, except those used topically for glaucoma. For each dispensing, we calculated the number of daily defined doses by multiplying the number of tablets dispensed by the dose per tablet in $\mathrm{mg}$, and dividing by the daily defined dose in $\mathrm{mg}$ from the World Health Organisation database [33].

Deaths were determined from the underlying cause of death in the regional breast cancer registries and National Mortality Collection, with ICD codes C50.0 to C50.9 classified as deaths from breast cancer.

\section{Confounders}


Demographic and clinical information came from the regional breast cancer registries, and covariates considered included date of diagnosis, age, ethnic group [34, 35], socioeconomic deprivation [36], urban/rural status [37], public/private status of the treatment facility, register, stage [38], grade [39], mode of detection (screen detected vs symptomatic), lymphovascular invasion, and receptor status (as defined previously [40], including Luminal A, Luminal B, Luminal B HER2+, HER2+ non-luminal, and triple negative). Other post-diagnostic medications included statins, aspirin and other non-steroidal antiinflammatory medications (NSAIDs), angiotensin converting enzyme inhibitors (ACEls), angiotensin receptor blockers (ARBs), and diuretics. Comorbidities adjusted for included any cardiac condition (angina, arrhythmia, congestive heart failure, hypertension, myocardial infarction, 'other cardiac conditions', and valve disease) as yes/no, diabetes, stroke, chronic obstructive pulmonary disorder, and peripheral vascular disease. We defined comorbidities as any of the above conditions appearing in a patient's linked hospital record (inpatient admissions) in the 5-year period before their breast cancer diagnosis.

\section{Statistical analyses}

Comparisons by BB use at baseline (date of diagnosis of breast cancer) were conducted using the chisquare test. We used Cox proportional hazard models to assess hazard ratios (HRs) of breast cancerspecific mortality associated with post-diagnostic BB use vs non-use. Death registrations and Pharmaceutical Collective coverage were complete to the end of 2017, so we followed patients from their breast cancer diagnosis until death or 31 December 2017. Women with no death recorded prior to 31 December 2017 were assumed to be alive as at 31 December 2017. Medication use was conceptualised as a time-varying covariate, such that time before the first dispensing was counted as 'nonuser' time, and time from the first dispensing to end of follow up was counted as 'user' time [41]. Models were adjusted in a systematic fashion, with the first adjustment including demographic and breast cancer clinical data, and the second adding other medication use and comorbidities.

Analyses were conducted considering BB use as a binary variable (user/nonuser), and also by splitting BB use into seven categories based on the number of daily defined doses (DDDs: categorised as 1-90 DDDs, 91-181 DDDs, 182-272 DDDs, 273-364 DDDs, 365-729 DDDs, 730-1094 DDDs, or 1095 or more DDDs, corresponding to the equivalent of 0-3 months, 3-6 months, 6-9 months, 9 months-1 year, 1-2 years, 2-3 years, and $3+$ years of BB use respectively). Dose analyses were conducted using a time varying approach, such that women spent time in the lowest category before moving into the next dose category. In order to compare patterns of risk observed for BBs to those of another cardiovascular medication with similar indications [42], the same analyses were carried out for calcium channel blockers.

To examine the effect of BB use in early-stage patients only, an analysis was carried out restricted to patients with stage 1 , stage 2 , or stage 3 a cancers. In this analysis, patients with an 'unknown' stage were excluded.

To evaluate the effect of the competing risk of death from other causes, the proportional subhazards model was also used [43]. For this analysis, all deaths apart from breast cancer deaths were treated as 
competing events.

As dispensings toward the end of life may reflect changes in morbidity (including cancer recurrence/progression) or in health care related to end of life care [44,45], we also conducted analyses lagging medication times [46]. In these analyses, patients are initially considered nonusers and then users after a lag period has elapsed after their first medication dispensing. Using this approach, dispensings toward the end of life are removed by the lag; for example, a 6-month lag will ignore dispensings in the 6 months prior to death/last follow up and classify these women are as medication nonusers as opposed to users. To appropriately account for different periods in which end of life care may be administered, we also considered lag periods of 1 year and 2 years. In these analyses, all medications were modelled in the same fashion (for example, if BBs were lagged by 6-months, all other medications were as well).

In order to compare BB users to patients using other medications for a similar indication, a further analysis was carried out comparing BB users to BB nonusers who used another antihypertensive medication. For this comparison, other antihypertensives included 'Potassium Sparing Combination Diuretics', 'Thiazide and Related Diuretics', 'ACE Inhibitors', 'ACE Inhibitors with Diuretics', 'Angiotensin II Antagonists', 'Angiotensin II Antagonists with Diuretics', 'Dihydropyridine Calcium Channel Blockers', 'Other Calcium Channel Blockers', 'Alpha Adrenoceptor Blockers', and 'Centrally-Acting Agents'. In this analysis, BB nonusers who used another antihypertensive were followed from their first post-diagnostic antihypertensive dispensing until death or 31 December 2017.

We also conducted an analysis with breast cancer recurrence (BCR) as the outcome. In this analysis, we defined a BCR as either a local/regional recurrence or distant metastasis and restricted the cohort to patients with early-stage breast cancer as above. Recurrences were determined from the breast cancer registry data through patient's routine clinical records, and women were followed from their breast cancer diagnosis until BCR, death, last follow up date, or end of Pharmaceutical Collection coverage (31 December 2017), whichever came first. These analyses examined risk of BCR associated with BB use vs non-use, as well as risk associated with different doses of BB use vs non-use.

Results are reported as HRs and their $95 \%$ confidence intervals (Cls), with the two-sided significance level set at 0.05. Statistical analyses were conducted in STATA 13.1 (StataCorp, College Station, TX).

\section{Results}

Median follow up for our cohort of 14,976 women was 4.51 years (range 0.01-10.99 years), with 1,341 dying of breast cancer, and 884 dying from other causes. Of these 14,976 women, $21 \%$ were dispensed a BB after diagnosis (Table 1). Higher proportions of BB users compared to nonusers were diagnosed in earlier years of the study period, were older, were from more deprived areas, and were treated in a public facility. A higher proportion of BB users than nonusers were also more likely to have used other medications (statins, aspirin, ACEls, ARBs, and diuretics) and to have had documented comorbidities (any cardiac condition, diabetes, stroke, COPD, and peripheral vascular disease) (all statistically significant differences, $p<0.05)$. 
Table 1

Characteristics of breast cancer patients by beta blocker use.

\begin{tabular}{|c|c|c|}
\hline \multirow[t]{2}{*}{ Characteristics } & \multicolumn{2}{|c|}{ Beta blocker use after diagnosis } \\
\hline & Ever $(n, \%)$ & Never $(n, \%)$ \\
\hline Overall & 3,195 & 11,781 \\
\hline \multicolumn{3}{|c|}{ Year of diagnosis } \\
\hline 2007-2008 & $534(17)$ & $1,393(12)$ \\
\hline 2009-2010 & $617(19)$ & 2,039 (17) \\
\hline 2011-2012 & $723(23)$ & $2,546(22)$ \\
\hline 2013-2014 & $693(22)$ & $2,823(24)$ \\
\hline 2015-2016 & $628(20)$ & $2,980(25)$ \\
\hline \multicolumn{3}{|l|}{ Age at diagnosis } \\
\hline$<50$ & $323(10)$ & $3,933(33)$ \\
\hline $50-59$ & 609 (19) & $3,389(29)$ \\
\hline $60-69$ & $1,038(32)$ & $2,789(24)$ \\
\hline 70-79 & $706(22)$ & $1,072(9)$ \\
\hline $80+$ & $519(16)$ & $598(5)$ \\
\hline \multicolumn{3}{|l|}{ Ethnic group } \\
\hline European & $2,425(76)$ & $8,603(73)$ \\
\hline Maori & $325(10)$ & $1,103(9)$ \\
\hline Pacific & $187(6)$ & $761(6)$ \\
\hline Asian & $182(6)$ & $1,033(9)$ \\
\hline Other & $66(2)$ & $281(2)$ \\
\hline \multicolumn{3}{|l|}{ Deprivation } \\
\hline $1-2$ & $431(13)$ & $2,208(19)$ \\
\hline $3-4$ & $576(18)$ & $2,345(20)$ \\
\hline
\end{tabular}

${ }^{a}$ Comorbidities included those in a patient's hospital records five years before breast cancer diagnosis. Cardiac conditions included any of angina, arrhythmia, congestive heart failure, hypertension, myocardial infarction, 'other cardiac conditions', and valve disease.

Note) The chi square test was statistically significant $(p<0.05)$ for every variable except for urban/rural. 


\begin{tabular}{|c|c|c|}
\hline \multirow[t]{2}{*}{ Characteristics } & \multicolumn{2}{|c|}{ Beta blocker use after diagnosis } \\
\hline & Ever $(n, \%)$ & Never $(n, \%)$ \\
\hline $5-6$ & $685(21)$ & $2,319(20)$ \\
\hline 7-8 & $513(16)$ & $1,744(15)$ \\
\hline $9-10$ & $571(18)$ & $1,643(14)$ \\
\hline Unknown & 419 (13) & $1,522(13)$ \\
\hline \multicolumn{3}{|l|}{ Urban/rural } \\
\hline Urban & $2,551(80)$ & $9,352(79)$ \\
\hline Rural & $227(7)$ & $914(8)$ \\
\hline Unknown & $417(13)$ & $1,515(13)$ \\
\hline \multicolumn{3}{|c|}{ Status of facility } \\
\hline Public & $2,333(73)$ & $7,594(64)$ \\
\hline Private & $862(27)$ & $4,187(36)$ \\
\hline \multicolumn{3}{|l|}{ Register } \\
\hline Auckland & $1,675(52)$ & $6,581(56)$ \\
\hline Christchurch & $506(16)$ & $1,918(16)$ \\
\hline Waikato & $607(19)$ & $1,638(14)$ \\
\hline Wellington & $407(13)$ & $1,644(14)$ \\
\hline \multicolumn{3}{|l|}{ Cancer stage } \\
\hline 1 & $1,378(43)$ & $5,368(46)$ \\
\hline 2 & $1,120(35)$ & $4,004(34)$ \\
\hline 3 & $397(12)$ & $1,533(13)$ \\
\hline 4 & $141(4)$ & $555(5)$ \\
\hline Unknown & $159(5)$ & $321(3)$ \\
\hline Cancer grade & & \\
\hline
\end{tabular}

${ }^{a}$ Comorbidities included those in a patient's hospital records five years before breast cancer diagnosis. Cardiac conditions included any of angina, arrhythmia, congestive heart failure, hypertension, myocardial infarction, 'other cardiac conditions', and valve disease.

Note) The chi square test was statistically significant $(p<0.05)$ for every variable except for urban/rural. 


\begin{tabular}{|c|c|c|}
\hline \multirow[t]{2}{*}{ Characteristics } & \multicolumn{2}{|c|}{ Beta blocker use after diagnosis } \\
\hline & Ever $(n, \%)$ & Never $(n, \%)$ \\
\hline Well differentiated & $660(21)$ & $2,613(22)$ \\
\hline Moderately differentiated & $1,496(47)$ & $5,061(43)$ \\
\hline Poorly differentiated & $859(27)$ & $3,455(29)$ \\
\hline Unknown & $180(6)$ & $652(6)$ \\
\hline \multicolumn{3}{|l|}{ Method of diagnosis } \\
\hline Symptomatic & $1,953(61)$ & $6,815(58)$ \\
\hline Screen detected & $1,242(39)$ & $4,966(42)$ \\
\hline \multicolumn{3}{|l|}{ Lymphovascular invasion } \\
\hline No & $1,881(59)$ & $7,064(60)$ \\
\hline Yes & $1,050(33)$ & $4,023(34)$ \\
\hline Unknown & $264(8)$ & $694(6)$ \\
\hline \multicolumn{3}{|l|}{ Receptor status } \\
\hline HER2+ non-luminal & $168(5)$ & $756(6)$ \\
\hline Luminal A & $2,074(65)$ & $7,872(67)$ \\
\hline Luminal B & $116(4)$ & $274(2)$ \\
\hline Luminal B HER2+ & $246(8)$ & $1,079(9)$ \\
\hline Triple negative & $324(10)$ & $1,219(10)$ \\
\hline Unknown & $267(8)$ & $581(5)$ \\
\hline \multicolumn{3}{|c|}{ Other medication use after diagnosis } \\
\hline Statins & $1,667(52)$ & $2,393(20)$ \\
\hline NSAIDs & $1,951(61)$ & 8,377 (71) \\
\hline Aspirin & $1,744(55)$ & 2,137 (18) \\
\hline ACEls & $1,718(54)$ & $2,410(20)$ \\
\hline
\end{tabular}

${ }^{a}$ Comorbidities included those in a patient's hospital records five years before breast cancer diagnosis. Cardiac conditions included any of angina, arrhythmia, congestive heart failure, hypertension, myocardial infarction, 'other cardiac conditions', and valve disease.

Note) The chi square test was statistically significant $(p<0.05)$ for every variable except for urban/rural. 


\begin{tabular}{|c|c|c|}
\hline \multirow[t]{2}{*}{ Characteristics } & \multicolumn{2}{|c|}{ Beta blocker use after diagnosis } \\
\hline & Ever $(n, \%)$ & Never $(n, \%)$ \\
\hline ARBs & $612(19)$ & $741(6)$ \\
\hline Diuretics & $1,546(48)$ & $1,879(16)$ \\
\hline \multicolumn{3}{|l|}{ Hospitalised comorbidities ${ }^{a}$} \\
\hline Any cardiac condition & $740(23)$ & $539(5)$ \\
\hline Diabetes & $246(8)$ & $319(3)$ \\
\hline Stroke & $133(4)$ & $127(1)$ \\
\hline COPD & $70(2)$ & $138(1)$ \\
\hline Peripheral vascular disease & $46(1)$ & $24(0.2)$ \\
\hline \multicolumn{3}{|c|}{$\begin{array}{l}\text { a Comorbidities included those in a patient's hospital records five years before breast cance } \\
\text { diagnosis. Cardiac conditions included any of angina, arrhythmia, congestive heart failure, } \\
\text { hypertension, myocardial infarction, 'other cardiac conditions', and valve disease. }\end{array}$} \\
\hline \multicolumn{3}{|c|}{$\begin{array}{l}\text { Note) The chi square test was statistically significant }(p<0.05) \text { for every variable except for } \\
\text { urban/rural. }\end{array}$} \\
\hline
\end{tabular}

We compared the risk of BCD associated with BB use (vs non-use) after diagnosis (Table 2). In the unadjusted model, BB use was associated with an increased risk of $B C D$ (HR=1.49; 95\% Cl: 1.31-1.70). This increased risk was markedly reduced after adjustment for demographic and breast cancer clinical factors (HR=1.16; 1.01-1.33), and modestly reduced with further adjustment for other medication use and comorbidities, such that there was only a small and non-statistically significant increased risk in the fully adjusted model (HR=1.11;0.95-1.29). The fully adjusted HR was similar in the analysis restricted to patients with early-stage cancers only ( $H R=1.17 ; 0.94-1.46)$. A similar fully adjusted finding was noted when adjusting for the competing risk of death from other causes ( $H R=1.05 ; 0.88-1.26)$. Lagging $B B$ use by various lengths of time did not substantially alter the HR (Table 2). When adjusting for demographic variables, clinical variables, comorbidities, and other medication use in four stages, it was found that demographic variables were the strongest confounders of the association (Supplementary Table 1). 
Table 2

Associations of breast cancer specific survival with post-diagnostic use of beta blockers (vs non-use) in breast cancer patients, by total dose.

\begin{tabular}{|c|c|c|c|c|c|}
\hline $\begin{array}{l}\text { Medication Usage } \\
\text { After Diagnosis }\end{array}$ & $\begin{array}{l}\text { No. Breast } \\
\text { cancer } \\
\text { deaths }\end{array}$ & $\begin{array}{l}\text { No. } \\
\text { person- } \\
\text { years }\end{array}$ & $\begin{array}{l}\text { Unadjusted } \\
\text { HR (95\% Cl) }\end{array}$ & $\begin{array}{l}\text { Adjusteda } \\
\text { HR }(95 \% \mathrm{Cl})\end{array}$ & $\begin{array}{l}\text { Fully adjusted } \\
\text { HR }(95 \% \mathrm{Cl})\end{array}$ \\
\hline BB nonuser & 1,036 & 60,301 & 1.00 & 1.00 & 1.00 \\
\hline BB user & 305 & 12,487 & $\begin{array}{l}1.49(1.31- \\
1.70)\end{array}$ & $\begin{array}{l}1.16(1.01- \\
1.33)\end{array}$ & $\begin{array}{l}1.11(0.95- \\
1.29)\end{array}$ \\
\hline $\begin{array}{l}\text { BB nonuser, early } \\
\text { stage }^{\mathrm{C}}\end{array}$ & 446 & 54,962 & 1.00 & 1.00 & 1.00 \\
\hline BB user, early stage ${ }^{c}$ & 151 & 11,223 & $\begin{array}{l}1.58(1.32- \\
1.91)\end{array}$ & $\begin{array}{l}1.29(1.06- \\
1.58)\end{array}$ & $\begin{array}{l}1.17(0.94- \\
1.46)\end{array}$ \\
\hline $\begin{array}{l}\text { BB user, adjusting for } \\
\text { competing risks }\end{array}$ & 305 & 12,487 & $\begin{array}{l}1.43(1.26- \\
1.63)\end{array}$ & $\begin{array}{l}1.11(0.94- \\
1.31)\end{array}$ & $\begin{array}{l}1.05(0.88- \\
1.26)\end{array}$ \\
\hline BB user, 6-month lag & 252 & 10,946 & $\begin{array}{l}1.38(1.20- \\
1.59)\end{array}$ & $\begin{array}{l}1.06(0.92- \\
1.23)\end{array}$ & $\begin{array}{l}1.14(0.97- \\
1.35)\end{array}$ \\
\hline BB user, 1-year lag & 220 & 9,496 & $\begin{array}{l}1.41(1.21- \\
1.63)\end{array}$ & $\begin{array}{l}1.09(0.93- \\
1.28)\end{array}$ & $\begin{array}{l}1.22(1.03- \\
1.46)\end{array}$ \\
\hline BB user, 2-year lag & 131 & 6,965 & $\begin{array}{l}1.21(1.00- \\
1.46)\end{array}$ & $\begin{array}{l}0.94(0.77- \\
1.14)\end{array}$ & $\begin{array}{l}1.05(0.84- \\
1.31)\end{array}$ \\
\hline $\begin{array}{l}1-90 \text { DDDs (0-3 } \\
\text { months) }\end{array}$ & 109 & 3,201 & $\begin{array}{l}2.09(1.72- \\
2.55)\end{array}$ & $\begin{array}{l}1.55(1.26- \\
1.89)\end{array}$ & $\begin{array}{l}1.40(1.14- \\
1.73)\end{array}$ \\
\hline $\begin{array}{l}91-181 \text { DDDs (3-6 } \\
\text { months) }\end{array}$ & 45 & 1,782 & $\begin{array}{l}1.44(1.07- \\
1.94)\end{array}$ & $\begin{array}{l}1.24(0.91- \\
1.68)\end{array}$ & $\begin{array}{l}1.15(0.84- \\
1.57)\end{array}$ \\
\hline $\begin{array}{l}182-272 \text { DDDs (6-9 } \\
\text { months) }\end{array}$ & 31 & 1,225 & $\begin{array}{l}1.42(0.99- \\
2.04)\end{array}$ & $\begin{array}{l}1.26(0.87- \\
1.81)\end{array}$ & $\begin{array}{l}1.22(0.84- \\
1.77)\end{array}$ \\
\hline $\begin{array}{l}273-364 \text { DDDs ( } 9 \\
\text { months-1 year) }\end{array}$ & 27 & 1,001 & $\begin{array}{l}1.50(1.02- \\
2.20)\end{array}$ & $\begin{array}{l}1.19(0.81- \\
1.75)\end{array}$ & $\begin{array}{l}1.13(0.76- \\
1.68)\end{array}$ \\
\hline $\begin{array}{l}365-729 \text { DDDs ( } 1 \\
\text { year-2 years) }\end{array}$ & 53 & 2,325 & $\begin{array}{l}1.33(1.01- \\
1.76)\end{array}$ & $\begin{array}{l}1.01(0.76- \\
1.34)^{(0.7}\end{array}$ & $\begin{array}{l}0.95(0.71- \\
1.28)\end{array}$ \\
\hline $\begin{array}{l}730-1094 \text { DDDs ( } 2 \\
\text { years-3 years) }\end{array}$ & 21 & 1,180 & $\begin{array}{l}1.12(0.73- \\
1.73)\end{array}$ & $\begin{array}{l}0.76(0.49- \\
1.19)\end{array}$ & $\begin{array}{l}0.78(0.50- \\
1.22)\end{array}$ \\
\hline $\begin{array}{l}1095 \text { or more DDDs } \\
\text { (3 or more years) }\end{array}$ & 19 & 1,774 & $\begin{array}{l}0.81(0.51- \\
1.28)\end{array}$ & $\begin{array}{l}0.54(0.34- \\
0.86)\end{array}$ & $\begin{array}{l}0.54(0.34- \\
0.87)\end{array}$ \\
\hline
\end{tabular}




\begin{tabular}{|llllll|}
\hline $\begin{array}{l}\text { Medication Usage } \\
\text { After Diagnosis }\end{array}$ & $\begin{array}{l}\text { No. Breast } \\
\text { cancer } \\
\text { deaths }\end{array}$ & $\begin{array}{l}\text { No. } \\
\text { person- } \\
\text { years }\end{array}$ & $\begin{array}{l}\text { Unadjusted } \\
\mathrm{HR}(95 \% \mathrm{Cl})\end{array}$ & $\begin{array}{l}\text { Adjusteda } \\
\mathrm{HR}(95 \% \mathrm{Cl})\end{array}$ & $\begin{array}{l}\text { Fully adjusted }^{\mathrm{b}} \\
\mathrm{HR}(95 \% \mathrm{Cl})\end{array}$ \\
\hline
\end{tabular}

${ }^{b}$ Second adjustment controlled for the previous covariates as well as other drug use and hospitalised comorbidities (other drugs including statins, NSAIDs and aspirin, ACEIs, ARBs, and diuretics. Comorbidities including any cardiac condition as yes/no, diabetes, stroke, COPD, and peripheral vascular disease). Other drug covariates were modelled in the same fashion as beta blockers (except for dose analysis, in which other drugs were classified as user/nonuser and modelled as time varying covariates).

${ }^{c}$ Restricted to patients with stage 1, stage 2, or stage 3a cancers. Patients with an 'unknown' stage were excluded in this analysis.

${ }^{d} D D D$ s refer to daily defined doses.

${ }^{e}$ The $p$ value for linear trend for the fully adjusted dose analysis was 0.0005 .

In the dose analysis, the highest risk was observed during the initial 0-3 months of BB use (fully adjusted $H R=1.40 ; 1.14-1.73$, Table 2). The risk decreased but remained elevated for use up to a year, after which the risk was reduced with increasing duration of use, and a 46\% reduction in BCD was found in those who took a BB for the equivalent of 3 or more years ( $H R=0.54 ; 0.34-0.87)$. Excluding nonusers (i.e., among users only), the overall $p$ value for linear trend was 0.0005 . In the same analysis with calcium channel blockers as the exposure of interest (Table 3), use of less than 3 months was not associated with an elevated risk and risk did not consistently decrease with increasing duration of use among users ( $p$ for trend=0.2). 
Table 3

Associations of breast cancer specific survival with post-diagnostic use of calcium channel blockers (vs non-use) in breast cancer patients, by total dose.

\begin{tabular}{|c|c|c|c|c|c|}
\hline $\begin{array}{l}\text { Medication Usage } \\
\text { After Diagnosis }\end{array}$ & $\begin{array}{l}\text { No. Breast } \\
\text { cancer } \\
\text { deaths }\end{array}$ & $\begin{array}{l}\text { No. } \\
\text { person- } \\
\text { years }\end{array}$ & $\begin{array}{l}\text { Unadjusted } \\
\text { HR (95\% Cl) }\end{array}$ & $\begin{array}{l}\text { Adjusted }^{\mathrm{a}} \\
\mathrm{HR}(95 \% \mathrm{Cl})\end{array}$ & $\begin{array}{l}\text { Fully adjusted } \\
\text { HR }(95 \% \mathrm{Cl})\end{array}$ \\
\hline CCB nonuser & 1,100 & 61,265 & 1.00 & 1.00 & 1.00 \\
\hline CCB user & 241 & 11,523 & $\begin{array}{l}1.23(1.07- \\
1.41)\end{array}$ & $\begin{array}{l}0.94(0.81- \\
1.10)\end{array}$ & $\begin{array}{l}0.89(0.76- \\
1.04)\end{array}$ \\
\hline $\begin{array}{l}1-90 \text { DDDs }(0-3 \\
\text { months) }\end{array}$ & 40 & 1,750 & $\begin{array}{l}1.34(0.98- \\
1.84)\end{array}$ & $\begin{array}{l}0.82(0.59- \\
1.14)\end{array}$ & $\begin{array}{l}0.83(0.60- \\
1.16)\end{array}$ \\
\hline $\begin{array}{l}91-181 \text { DDDs (3-6 } \\
\text { months) }\end{array}$ & 25 & 1,121 & $\begin{array}{l}1.32(0.88- \\
1.96)\end{array}$ & $\begin{array}{l}0.97(0.65- \\
1.45)\end{array}$ & $\begin{array}{l}0.88(0.59- \\
1.32)\end{array}$ \\
\hline $\begin{array}{l}182-272 \text { DDDs (6-9 } \\
\text { months) }\end{array}$ & 30 & 882 & $\begin{array}{l}1.89(1.31- \\
2.72)\end{array}$ & $\begin{array}{l}1.51(1.05- \\
2.19)\end{array}$ & $\begin{array}{l}1.47(1.02- \\
2.14)\end{array}$ \\
\hline $\begin{array}{l}\text { 273-364 DDDs (9 } \\
\text { months-1 year) }\end{array}$ & 23 & 837 & $\begin{array}{l}1.50(0.99- \\
2.27)\end{array}$ & $\begin{array}{l}1.26(0.83- \\
1.92)\end{array}$ & $\begin{array}{l}1.18(0.77- \\
1.80)\end{array}$ \\
\hline $\begin{array}{l}365-729 \text { DDDs ( } 1 \\
\text { year-2 years) }\end{array}$ & 44 & 2,257 & $\begin{array}{l}1.05(0.77- \\
1.42)\end{array}$ & $\begin{array}{l}0.83(0.61- \\
1.12)\end{array}$ & $\begin{array}{l}0.78(0.57- \\
1.06)\end{array}$ \\
\hline $\begin{array}{l}730-1094 \text { DDDs ( } 2 \\
\text { years-3 years) }\end{array}$ & 30 & 1,419 & $\begin{array}{l}1.16(0.81- \\
1.68)\end{array}$ & $\begin{array}{l}0.95(0.65- \\
1.37)\end{array}$ & $\begin{array}{l}0.88(0.60- \\
1.28)\end{array}$ \\
\hline $\begin{array}{l}1095 \text { or more DDDs } \\
\text { (3 or more years) }\end{array}$ & 49 & 3,258 & $\begin{array}{l}1.00(0.75- \\
1.34)\end{array}$ & $\begin{array}{l}0.83(0.62- \\
1.12)\end{array}$ & $\begin{array}{l}0.74(0.55- \\
1.01)\end{array}$ \\
\hline \multicolumn{6}{|c|}{$\begin{array}{l}\text { a First adjustment controlled for date of } d x \text {, age, ethnic group, deprivation, urban/rural status, } \\
\text { public/private status of the facility, register, stage, grade, mode of detection, lymphovascular invasion, } \\
\text { and receptor status. }\end{array}$} \\
\hline \multicolumn{6}{|c|}{$\begin{array}{l}{ }^{b} \text { Second adjustment controlled for the previous covariates as well as other drug use and hospitalised } \\
\text { comorbidities (other drugs including statins, NSAIDs and aspirin, ACEIS, ARBS, and diuretics. } \\
\text { Comorbidities including any cardiac condition as yes/no, diabetes, stroke, COPD, and peripheral } \\
\text { vascular disease). Other drug covariates were modelled in the same fashion as beta blockers (except } \\
\text { for dose analysis, in which other drugs were classified as user/nonuser and modelled as time varying } \\
\text { covariates). }\end{array}$} \\
\hline \multicolumn{6}{|c|}{${ }^{c} D D D$ s refer to daily defined doses. } \\
\hline
\end{tabular}

When comparing BB users to a BB nonuser group who used another antihypertensive (Table 4), a $25 \%$ increased risk of $B C D$ was found (fully adjusted $H R=1.25 ; 1.06-1.48$ ). This increased risk was reduced to a null effect after lagging medications by 2 years $(H R=0.99 ; 0.78-1.26)$. 
Table 4

Associations of breast cancer specific survival with post-diagnostic use of beta blockers (vs non-use) in breast cancer patients, using a comparison group of nonusers who used another antihypertensive.

\begin{tabular}{|c|c|c|c|c|c|}
\hline $\begin{array}{l}\text { Medication Usage After } \\
\text { Diagnosis }\end{array}$ & $\begin{array}{l}\text { No. Breast } \\
\text { cancer } \\
\text { deaths }\end{array}$ & $\begin{array}{l}\text { No. } \\
\text { Person- } \\
\text { years }\end{array}$ & $\begin{array}{l}\text { Unadjusted } \\
\text { HR (95\% Cl) }\end{array}$ & $\begin{array}{l}\text { Adjusteda } \\
\mathrm{HR}(95 \% \\
\mathrm{Cl})\end{array}$ & $\begin{array}{l}\text { Fully } \\
\text { adjusted }{ }^{\mathrm{b}} \mathrm{HR} \\
(95 \% \mathrm{Cl})\end{array}$ \\
\hline $\begin{array}{l}\text { BB nonusers who used } \\
\text { another antihypertensive }\end{array}$ & 320 & 17,709 & 1.00 & 1.00 & 1.00 \\
\hline BB user & 305 & 12,487 & $\begin{array}{l}1.37(1.18- \\
1.61)\end{array}$ & $\begin{array}{l}1.21(1.03- \\
1.42)\end{array}$ & $\begin{array}{l}1.25(1.06- \\
1.48)\end{array}$ \\
\hline BB user, 6-month lag & 252 & 10,946 & $\begin{array}{l}1.34(1.13- \\
1.60)\end{array}$ & $\begin{array}{l}1.20(1.00- \\
1.43)\end{array}$ & $\begin{array}{l}1.23(1.02- \\
1.47)\end{array}$ \\
\hline BB user, 1-year lag & 220 & 9,496 & $\begin{array}{l}1.38(1.15- \\
1.66)\end{array}$ & $\begin{array}{l}1.26(1.04- \\
1.52)\end{array}$ & $\begin{array}{l}1.30(1.07- \\
1.58)\end{array}$ \\
\hline BB user, 2-year lag & 131 & 6,965 & $\begin{array}{l}1.06(0.85- \\
1.33)\end{array}$ & $\begin{array}{l}0.96(0.77- \\
1.22)\end{array}$ & $\begin{array}{l}0.99(0.78- \\
1.26)\end{array}$ \\
\hline \multicolumn{6}{|c|}{$\begin{array}{l}\text { a First adjustment controlled for date of } d x \text {, age, ethnic group, deprivation, urban/rural status, } \\
\text { public/private status of the facility, register, stage, grade, mode of detection, lymphovascular invasion } \\
\text { and receptor status. }\end{array}$} \\
\hline
\end{tabular}

In the analysis considering recurrence as the outcome (Table 5), there was no statistically significant association found between $B B$ use and $B C R(H R=1.06 ; 0.90-1.26)$. In the dose analysis, there was no longer an elevated risk associated with the initial 0-3 months of use (fully adjusted $H R=1.13 ; 0.87-1.49$, Table 5). The risk was inconsistent across dose categories, however there was a trend of a reduced risk with increasing duration of use among users ( $p$ for trend $=0.0065$ ). 
Table 5

Associations of breast cancer recurrence with post-diagnostic use of beta blockers (vs non-use) in breast cancer patients, by total dose.

\begin{tabular}{|c|c|c|c|c|c|}
\hline $\begin{array}{l}\text { Medication Usage } \\
\text { After Diagnosis }\end{array}$ & $\begin{array}{l}\text { No. Breast } \\
\text { cancer } \\
\text { recurrences }\end{array}$ & $\begin{array}{l}\text { No. } \\
\text { person- } \\
\text { years }\end{array}$ & $\begin{array}{l}\text { Unadjusted } \\
\text { HR (95\% Cl) }\end{array}$ & $\begin{array}{l}\text { Adjusted }^{a} \\
\text { HR }(95 \% \\
\text { Cl) }\end{array}$ & $\begin{array}{l}\text { Fully adjusted } \\
\text { HR }(95 \% \mathrm{Cl})\end{array}$ \\
\hline BB nonuser & 989 & 47,349 & 1.00 & 1.00 & 1.00 \\
\hline BB user & 227 & 9,619 & $\begin{array}{l}1.12(0.97- \\
1.29)\end{array}$ & $\begin{array}{l}1.02(0.87- \\
1.19)\end{array}$ & $\begin{array}{l}1.06(0.90- \\
1.26)\end{array}$ \\
\hline $\begin{array}{l}1-90 \text { DDDs ( } 0-3 \\
\text { months) }\end{array}$ & 109 & 2,454 & $\begin{array}{l}1.24(0.96- \\
1.62)\end{array}$ & $\begin{array}{l}1.10(0.85- \\
1.44)\end{array}$ & $\begin{array}{l}1.13(0.87- \\
1.49)\end{array}$ \\
\hline $\begin{array}{l}91-181 \text { DDDs (3-6 } \\
\text { months) }\end{array}$ & 45 & 1,391 & $\begin{array}{l}1.36(0.99- \\
1.85)\end{array}$ & $\begin{array}{l}1.29(0.94- \\
1.77)\end{array}$ & $\begin{array}{l}1.33(0.96- \\
1.83)\end{array}$ \\
\hline $\begin{array}{l}182-272 \text { DDDs (6-9 } \\
\text { months) }\end{array}$ & 31 & 950 & $\begin{array}{l}1.13(0.76- \\
1.68)\end{array}$ & $\begin{array}{l}1.06(0.71- \\
1.59)\end{array}$ & $\begin{array}{l}1.10(0.73- \\
1.66)\end{array}$ \\
\hline $\begin{array}{l}273-364 \text { DDDs (9 } \\
\text { months-1 year) }\end{array}$ & 27 & 777 & $\begin{array}{l}1.08(0.69- \\
1.70)\end{array}$ & $\begin{array}{l}0.97(0.61- \\
1.53)\end{array}$ & $\begin{array}{l}1.00(0.63- \\
1.59)\end{array}$ \\
\hline $\begin{array}{l}365-729 \text { DDDs ( } 1 \\
\text { year-2 years) }\end{array}$ & 53 & 1,775 & $\begin{array}{l}1.30(0.98- \\
1.72)\end{array}$ & $\begin{array}{l}1.16(0.87- \\
1.55)\end{array}$ & $\begin{array}{l}1.21(0.90- \\
1.62)\end{array}$ \\
\hline $\begin{array}{l}730-1094 \text { DDDs ( } 2 \\
\text { years-3 years) }\end{array}$ & 21 & 928 & $\begin{array}{l}0.61(0.35- \\
1.09)\end{array}$ & $\begin{array}{l}0.54(0.31- \\
0.96)\end{array}$ & $\begin{array}{l}0.57(0.32- \\
1.01)\end{array}$ \\
\hline $\begin{array}{l}1095 \text { or more DDDs } \\
\text { (3 or more years) }\end{array}$ & 19 & 1,343 & $\begin{array}{l}0.67(0.41- \\
1.09)\end{array}$ & $\begin{array}{l}0.62(0.38- \\
1.02)\end{array}$ & $\begin{array}{l}0.65(0.39- \\
1.07)\end{array}$ \\
\hline
\end{tabular}

${ }^{a}$ First adjustment controlled for date of $d x$, age, ethnic group, deprivation, urban/rural status, public/private status of the facility, register, stage, grade, mode of detection, lymphovascular invasion, and receptor status.

${ }^{b}$ Second adjustment controlled for the previous covariates as well as other drug use and hospitalised comorbidities (other drugs including statins, NSAIDs and aspirin, ACEIs, ARBs, and diuretics.

Comorbidities including any cardiac condition as yes/no, diabetes, stroke, COPD, and peripheral vascular disease). Other drug covariates were modelled in the same fashion as beta blockers (except for dose analysis, in which other drugs were classified as user/nonuser and modelled as binary time varying covariates).

${ }^{c}$ Restricted to patients with stage 1, stage 2, or stage 3a cancers. Patients with an 'unknown'stage were excluded in this analysis.

${ }^{d} D D D$ s refer to daily defined doses.

${ }^{e}$ The $p$ value for linear trend for the fully adjusted dose analysis was 0.0065 . 
There was a small and non-statistically significant increased risk between any BB use after breast cancer diagnosis and BCD in this large NZ population-based study of breast cancer patients after adjustment for demographic and clinical factors, comorbidities, and other medication use. However, further analyses revealed a complex pattern: there was an increased risk associated with the initial few months of use, but a decreasing risk with longer term use, with evidence of a dose-response trend indicative of a potential protective effect of long-term BB use on BCD.

Our primary finding is consistent with a number of previous studies indicating no significant association between BB use and BCD in their fully adjusted analyses [16, 20,22-25], while a number have found BBs to be protective [16-18], and one to increase the risk of BCD [26]. For example, a study of 466 breast cancer patients in England found hypertensive patients treated with BBs to have a $71 \%$ reduction in BCD $(H R=0.29 ; 0.12-0.71)$ [17]. Conversely, a study of 14,766 breast cancer patients aged between 66 and 80 in the United States found BBs to increase the risk of BCD by $41 \%(H R=1.41 ; 1.07-1.84)$ [26]. Another English study of 9,817 breast cancer patients found BB use to be associated with a smaller and not statistically significant increased risk of $B C D(H R=1.20 ; 0.92-1.57)$ [22]. All these studies considered $B B$ use as a time-varying covariate. One other study indicated no association between BB use and BCD [21], and one found BBs to be protective [19], however these two studies did not account for the time-varying nature of medication use.

There are many reasons why results of studies examining medication use and cancer outcomes may vary. For example, studies which do not account for the time-varying nature of medication use are more likely to observe a protective effect through the introduction of immortal time bias [19, 21, 28, 41]. Furthermore, the definition of medication use varies from study to study, with some considering BB use prior to diagnosis, some after diagnosis, and some in other periods. In our study, we only considered BB use after diagnosis, arguably the most clinically relevant exposure period. Indications for BBs have also changed over time, and heart failure is now their most common indication, whereas BBs were commonly prescribed for hypertension 15-20 years ago $[9,47]$. The general health of patients medicated with BBs has therefore likely changed from a healthier group to a less healthy group over time, with corresponding implications for their risk of BCD.

We found the risk of BCD to decrease with increasing doses of BBs ( $p$ for trend=0.0005), with a HR of 1.40 (1.14-1.73) for the initial 0 to 3 months of use, and a HR of 0.54 (0.34-0.87) for 3 or more years of use. After the equivalent of a year's use, the risk was decreased with increasing duration of use, which may be suggestive of a protective effective associated with long-term BB use. Previous literature investigating dose-response effects of BBs has been sparse, however our results are in contrast to two previous papers (although neither paper reported a statistically significant dose effect) [20, 22], while one found no pattern [26], and one also found a similar pattern to ours for a non-selective BB, propranolol [16]. To our knowledge, ours is the first paper to study the long-term (i.e., 3 years+) effect of BBs on BCD and find a consistent dose-response pattern indicating a protective effect associated with long-term use. It is possible that this relationship can partly be explained by a healthy user effect [48], whereby those who tolerated BBs well and were healthier in general were more likely to stay on the medication for longer 
periods. However, the fact that this pattern was not reproduced for other cardiovascular medications (e.g., calcium channel blockers, Table 3) only serves to strengthen the suggestion that there may indeed be a causal and protective relationship between long-term BB use and BCD. It seems plausible that the increased risk of BCD we observed in the initial period of use (between 0 and 3 months) can predominantly be explained by women being medicated for symptomatic conditions toward the end of life. These women then go on to die soon after and are coded as dying from breast cancer. It might be considered that this end-of-life dispensing is creating a spurious dose-response effect for BBs, however a statistically significant $p$ for trend value was observed even after excluding these users from the analysis (Supplementary Table 2). The protective effect observed in long-term users can likely be explained by the absence of any 'end of life' prescribing effect, as well as an increasing systemic uptake of BBs over time, with higher dosings seemingly necessary to elicit any real protective effect.

The hypothesis that increased risk associated with the first few months of use is driven by short-term use toward the end of life is supported by an amalgamation of evidence. Firstly, there was a clear pattern in women's median time to death/last follow up from their first BB dispensing by dose category: those who took BBs in lower doses consistently had a shorter time interval between their first dispensing and death/last follow up than those who took BBs for longer (Supplementary Table 3). Secondly, there was no longer a statistically significant increased risk in short-term users when considering BCR as the outcome (Table 5). This supports the idea that a significant number of women are dispensed a BB after a recurrence (perhaps because of complications associated with the recurrence), and then go on to die soon after. Lastly, the short-term use category is the dose category with the highest proportion of 'new' users (new users meaning those who did not have a BB dispensing in the year prior to diagnosis) relative to any other dose category (Supplementary Table 4). This is consistent with their first dispensing being toward the end of life, rather than continuing use initiated prior to their breast cancer diagnosis. Furthermore, the short-term user category had the highest proportion of breast cancer deaths relative to any other dose category.

It has been suggested that breast cancer and CVD deaths are sometimes misclassified, with one study suggesting that women's cancer diagnosis is often perceived as the overriding medical priority in patients with both cancer and CVD [49]. However, we found similar associations between BBs and BCD and BBs and $B C R$, which argues against misclassification of cause of death as a major issue in our cohort (as most women who die from breast cancer have had a BCR [50]). Furthermore, evidence from an Australian study assessing the accuracy of national cause of death coding found the specificity of cancer deaths to be $99.2 \%$ [51], showing that very few deaths were wrongly attributed to cancer.

When BB users were compared with a more similar comparison group (BB nonusers who used another antihypertensive), we observed a $25 \%$ increased risk of BCD after adjustment for confounding variables. This result may be explained by BB dispensings toward the end of life, as the HR decreased from 1.25 (1.06-1.48) to 0.99 (0.78-1.26) when lagging medication times by 2 years. Therefore, it appears that any differences in the HRs between different comparison groups can mostly be attributed to differences in 
end-of-life care between the two groups, rather than any fundamental differences in their underlying characteristics.

Finally, adjusting for the competing risk of death from other causes or analysing early-stage patients only did not substantially affect the HR. Moreover, when we applied these types of analyses to our other results, the HR did not change to a degree that would alter the interpretation of our results.

The primary strength of our study is that we had a large cohort of breast cancer patients followed up over a relatively long time period sourced from four population-based databases. The Auckland and Waikato databases have been checked against the National Cancer Registry and found to be at least $99 \%$ complete, and the registry data we used contains more comprehensive and accurate information than the national data sources $[32,52,53]$. Our pharmaceutical data was derived from a high quality and automated national database, and there was no recall bias [54] associated with medication records as a result. Furthermore, unlike many other countries, New Zealand records medication dispensings instead of prescriptions, which are a stronger proxy for medication adherence. We also conceptualised medication use as time varying covariates, and therefore avoided the introduction of immortal time bias that invariably biases results in favour of the medication [41].

Our study also has limitations. We did not have access to primary care data, which meant that our comorbidity data was restricted to hospital admissions in the relevant timeframe. Furthermore, this limited access to a range of potential confounders such as body mass index, alcohol intake, and smoking status, all of which would generally be available through general practitioner records. However, these limitations in residual confounders were somewhat mitigated by the use of a more balanced comparison group. The most commonly prescribed BB in New Zealand is a selective BB, metoprolol [9], which was used by $78 \%$ of the BB users in our study. Therefore, we did not have the power to explore the relationship between non-selective BBs (such as propranolol) and BCD. Several preclinical studies have suggested that non-selective BBs may have a higher efficacy in inhibiting pathways involved in breast cancer progression and metastasis $[15,55]$. Finally, we did not adjust for breast cancer treatment such as chemotherapy and radiotherapy. However, it is likely that any treatment variables would have been highly correlated with other variables in our model such as stage and grade.

In conclusion, there was only a weak and non-significant association between post-diagnostic BB use and BCD in this large population-based study of NZ breast cancer patients. Further analyses showed an increased risk associated with the first few months of use, likely to be due to use toward the end of life. For longer-term use, risk of death decreased with increasing duration of use, suggesting that long-term BB use may confer a protective effect on BCD. Further research is warranted to assess if this relationship is replicated in other clinical settings.

\section{Declarations}

Acknowledgements 
We would like to thank the Auckland Medical Research Foundation for providing funding to carry out this study. We would also like to thank Chris Cardwell (Queen's University), Rod Jackson (University of Auckland), Roger Marshall (University of Auckland), and Bruce Arroll (University of Auckland) for their individual contributions to the paper.

\section{Funding}

Oliver Scott was supported by a Auckland Medical Research Foundation doctoral scholarship (Ref: 1217004).

This project was also supported by a Auckland Medical Research Foundation project grant (Ref: 1118017).

\section{Competing Interests}

The authors have no relevant financial or non-financial interests to disclose.

\section{Author Contributions}

OWS, STT, JME, and AC substantially contributed to the conception and design of the study, and interpreted data alongside LAH. OWS led the analysis, and STT, MK, IC, and RL led data collection. OWS wrote the manuscript, and all authors critically revised and approved the final manuscript.

\section{Data Availability.}

The datasets used in this study contain personal information and are not publicly available, but may be requested from the Breast Cancer Foundation New Zealand and the Ministry of Health (NZ).

\section{Ethics approval}

This study was approved by the Central Health and Disability Ethics Committee (Ref: 19/CEN/4).

\section{Consent to participate}

Not applicable.

\section{Consent for publication}

Not applicable.

\section{References}

1. Ghoncheh M, Pournamdar Z, Salehiniya $\mathrm{H}$. Incidence and mortality and epidemiology of breast cancer in the world. Asian Pac J Cancer Prev 2016; 17: 43-46. 
2. Land L, Dalton $S$, Jensen $M$ et al. Influence of comorbidity on the effect of adjuvant treatment and age in patients with early-stage breast cancer. Br J Cancer 2012; 107: 1901-1907.

3. Mehta LS, Watson KE, Barac A et al. Cardiovascular disease and breast cancer: where these entities intersect: a scientific statement from the American Heart Association. Circulation 2018; 137: e30-e66.

4. Ministry of Health. (2019). Annual Update of Key Results 2018/19: New Zealand Health Survey. Wellington: Ministry of Health.

5. Ford ES, Li C, Zhao G et al. Trends in low-risk lifestyle factors among adults in the United States: findings from the Behavioral Risk Factor Surveillance System 1996-2007. Prev Med 2010; 51: 403407.

6. Ram CVS. Beta-blockers in hypertension. Am J Cardiol 2010; 106: 1819-1825.

7. Aronow WS. Current role of beta-blockers in the treatment of hypertension. Expert Opin Pharmacother 2010; 11: 2599-2607.

8. Jackson R, Barham P, Bills $\mathrm{J}$ et al. Management of raised blood pressure in New Zealand: a discussion document. Br Med J 1993; 307: 107-110.

9. Best Practice Advocacy Centre New Zealand. Beta-blockers for cardiovascular conditions: one size does not fit all patients. https://bpac.org.nz/2017/beta-blockers.aspx(Date Accessed. 2017 Accessed, date last accessed)

10. Bangalore S, Messerli FH, Kostis JB et al. Cardiovascular protection using beta-blockers: a critical review of the evidence. J Am Coll Cardiol 2007; 50: 563-572.

11. Vandewalle B, Revillion F, Lefebvre J. Functional $\beta$-adrenergic receptors in breast cancer cells. J Cancer Res Clin Oncol 1990; 116: 303-306.

12. Cole SW, Sood AK. Molecular pathways: beta-adrenergic signaling in cancer. Clin Cancer Res 2012; 18: 1201-1206.

13. Tilan J, Kitlinska J. Sympathetic neurotransmitters and tumor angiogenesis-link between stress and cancer progression. J Oncol 2010; 2010.

14. Vaklavas C, Chatzizisis YS, Tsimberidou AM. Common cardiovascular medications in cancer therapeutics. Pharmacol Ther 2011; 130: 177-190.

15. Sloan EK, Priceman SJ, Cox BF et al. The sympathetic nervous system induces a metastatic switch in primary breast cancer. Cancer Res 2010; 70: 7042-7052.

16. Barron TI, Connolly RM, Sharp L et al. Beta blockers and breast cancer mortality: a population-based study. J Clin Oncol 2011; 29: 2635-2644.

17. Powe DG, Voss MJ, Zänker KS et al. Beta-blocker drug therapy reduces secondary cancer formation in breast cancer and improves cancer specific survival. Oncotarget 2010; 1: 628.

18. Botteri $\mathrm{E}$, Munzone $\mathrm{E}$, Rotmensz $\mathrm{N}$ et al. Therapeutic effect of $\beta$-blockers in triple-negative breast cancer postmenopausal women. Breast Cancer Res Treat 2013; 140: 567-575.

19. Chae YK, Brown EN, Lei $X$ et al. Use of ACE inhibitors and angiotensin receptor blockers and primary breast cancer outcomes. J Cancer 2013; 4: 549. 
20. Ganz PA, Habel LA, Weltzien EK et al. Examining the influence of beta blockers and ACE inhibitors on the risk for breast cancer recurrence: results from the LACE cohort. Breast Cancer Res Treat 2011; 129: 549.

21. Holmes MD, Hankinson SE, Feskanich D et al. Beta blockers and angiotensin-converting enzyme inhibitors' purported benefit on breast cancer survival may be explained by aspirin use. Breast Cancer Res Treat 2013; 139: 507-513.

22. Cardwell CR, Coleman HG, Murray LJ et al. Beta-blocker usage and breast cancer survival: a nested case-control study within a UK Clinical Practice Research Datalink cohort. Int J Epidemiol 2013; 42: 1852-1861.

23. Musselman RP, Bennett S, Li W et al. Association between perioperative beta blocker use and cancer survival following surgical resection. Eur J Surg Oncol 2018; 44: 1164-1169.

24. Santala EE, Murto MO, Artama M et al. Angiotensin Receptor Blockers Associated with Improved Breast Cancer Survival-A Nationwide Cohort Study from Finland. Cancer Epidemiol Biomarkers Prev 2020; 29: 2376-2382.

25. Cui Y, Wen W, Zheng T et al. Use of antihypertensive medications and survival rates for breast, colorectal, lung, or stomach cancer. Am J Epidemiol 2019; 188: 1512-1528.

26. Chen L, Chubak J, Boudreau DM et al. Use of Antihypertensive Medications and Risk of Adverse Breast Cancer Outcomes in a SEER-Medicare Population. Cancer Epidemiol Biomarkers Prev 2017; 26: 1603-1610.

27. Hiller JG, Cole SW, Crone EM et al. Preoperative $\beta$-blockade with propranolol reduces biomarkers of metastasis in breast cancer: a phase II randomized trial. Clin Cancer Res 2020; 26: 1803-1811.

28. Weberpals J, Jansen L, Carr PR et al. Beta blockers and cancer prognosis-the role of immortal time bias: a systematic review and meta-analysis. Cancer Treat Rev 2016; 47: 1-11.

29. Breast Cancer Foundation National Register. (2021). Data Entry Reference Guide. Wellington: Breast Cancer Foundation National Register.

30. Ministry of Health. (2017). Pharmaceutical Claims Data Mart (PHARMS). Wellington: Ministry of Health.

31. Ministry of Health. (2017). Mortality Collection Data Dictionary. Wellington: Ministry of Health.

32. Seneviratne S, Campbell I, Scott $\mathrm{N}$ et al. Accuracy and completeness of the New Zealand Cancer Registry for staging of invasive breast cancer. Cancer Epidemiol 2014; 38: 638-44. doi: 10.1016/j.canep.2014.06.008.

33. World Health Organization. WHO Collaborating Centre for Drug Statistics Methodology. https://www.whocc.no/(Date Accessed. 2019 Accessed, date last accessed)

34. Ministry of Health. Ethnicity code tables. http://www.health.govt.nz/nz-health-statistics/datareferences/code-tables/common-code-tables/ethnicity-code-tables.(Date Accessed. 2010 Accessed, date last accessed) 
35. Ministry of Health. (2004). Ethnicity Data Protocols for the Health and Disability Sector. Wellington: Ministry of Health.

36. Atkinson J, Salmond C, Crampton P. NZDep2013 index of deprivation. Wellington: Department of Public Health, University of Otago 2014.

37. Statistics New Zealand. (2007). New Zealand: An Urban/Rural Profile. Wellington: Statistics New Zealand.

38. Edge S, Byrd D, Compton C et al. American Joint Committee on Cancer cancer staging manual. Ann. Surg. Oncol 2010; 17: 1471-1474.

39. Elston CW, Ellis IO. Pathological prognostic factors in breast cancer. I. The value of histological grade in breast cancer: experience from a large study with long-term follow-up. Histopathology 1991; 19: 403-410.

40. Lawrenson R, Lao C, Campbell I et al. The impact of different tumour subtypes on management and survival of New Zealand women with Stage I-III breast cancer. N Z Med J 2018; 131: 51-60.

41. Lévesque LE, Hanley JA, Kezouh A et al. Problem of immortal time bias in cohort studies: example using statins for preventing progression of diabetes. Br Med J 2010; 340: b5087.

42. Neagoe A-M, Rexhaj E, Grossman E et al; Beta Blockers and Calcium Channel Blockers. Cardiovascular Hemodynamics. Springer, 2019, 73-88.

43. Fine JP, Gray RJ. A Proportional Hazards Model for the Subdistribution of a Competing Risk. J Am Stat Assoc 1999; 94: 496-509. doi: 10.1080/01621459.1999.10474144.

44. Adler ED, Goldfinger JZ, Kalman J et al. Palliative care in the treatment of advanced heart failure. Circulation 2009; 120: 2597-2606.

45. Davidson PM, Macdonald PS, Newton PJ et al. End stage heart failure patients: Palliative care in general practice. Aust Fam Physician 2010; 39: 916-920.

46. Chubak J, Boudreau DM, Wirtz HS et al. Threats to validity of nonrandomized studies of postdiagnosis exposures on cancer recurrence and survival. J Natl Cancer Inst 2013; 105: 14561462.

47. Wiysonge CS, Bradley HA, Volmink J et al. Beta-blockers for hypertension. Cochrane Database Syst Rev 2017.

48. Yola M, Lucien A. Evidence of the depletion of susceptibles effect in non-experimental pharmacoepidemiologic research. J Clin Epidemiol 1994; 47: 731-737.

49. Patnaik JL, Byers T, DiGuiseppi C et al. Cardiovascular disease competes with breast cancer as the leading cause of death for older females diagnosed with breast cancer: a retrospective cohort study. Breast Cancer Res 2011; 13: R64.

50. American Society of Clinical Oncology. Breast Cancer: Statistics. https://www.cancer.net/cancertypes/breast-cancer/statistics(Date Accessed. 2021 Accessed, date last accessed)

51. Magliano D, Liew D, Pater H et al. Accuracy of the Australian National Death Index: comparison with adjudicated fatal outcomes among Australian participants in the Long-term Intervention with 
Pravastatin in Ischaemic Disease (LIPID) study. Aust N Z J Public Health 2003; 27: 649-653.

52. Gurney J, Sarfati D, Dennett $E$ et al. The completeness of cancer treatment data on the National Health Collections. N Z Med J 2013; 126: 69-74.

53. Neave L, Harvey V, Benjamin C et al. The Auckland Breast Cancer Register: a special project of the Auckland Breast Cancer Study Group. N Z Med J 2003; 116: U648.

54. Glintborg B, Poulsen HE, Dalhoff KP. The use of nationwide on-line prescription records improves the drug history in hospitalized patients. Br J Clin Pharmacol 2008; 65: 265-269.

55. Lang K, Drell IV TL, Lindecke A et al. Induction of a metastatogenic tumor cell type by neurotransmitters and its pharmacological inhibition by established drugs. Int J Cancer 2004; 112: 231-238.

\section{Supplementary Files}

This is a list of supplementary files associated with this preprint. Click to download.

- Supplementarytablesnew.doc 